\title{
Ciudadanía, integración y universalismo: análisis y evaluación normativa de los programas de integración cívica en Alemania y Francia desde una perspectiva liberal
}

\author{
Citizenship, integration and universalism: \\ Normative analysis and evaluation of civic integration programmes \\ in Germany and France from a liberal perspective
}

\author{
CARMEN INNERARITY \\ Universidad Pública de Navarra
}

\section{Cómo citar/Citation}

Innerarity, C. (2016). Ciudadanía, integración y universalismo: análisis y evaluación normativa de los programas de integración cívica en Alemania y Francia desde una perspectiva liberal. Revista Española de Ciencia Política, 41, 39-66. Doi: http://dx.doi.org/10.21308/recp.41.02

\section{Resumen}

Este trabajo analiza el contenido de los cursos y exámenes de ciudadanía introducidos en los últimos años en Europa. La forma de entender la nación y la ciudadanía en cada país ¿̨determina la orientación de dichos programas o, por el contrario, encontramos ciertas similitudes entre ellos, independientemente de su idea de nación? ¿Cómo se explican las semejanzas y las diferencias? ¿Se refleja en los programas una idea de la pertenencia basada en el compromiso con valores cívicos -universalistas e integradores- y no tanto en tradiciones culturales compartidas -particularistas y excluyentes-? ¿O existen tensiones entre la propuesta normativa del universalismo liberal y su plasmación en ciertas políticas de integración?

Para contestar a estas preguntas, propongo un marco analítico que permite evaluar con criterios normativos los programas de integración cívica de Francia y Alemania. Para ello realizo un análisis de los programas en detalle, tratando de explicar sus similitudes y diferencias, tanto desde una perspectiva teórica como en relación a la vida política en cada uno de los dos países. A pesar de representar dos concepciones divergentes de nación y de ciudadanía, el análisis de los programas muestra no pocas similitudes entre ellos. Sin embargo, frente a lo que cabría esperar, en ambos casos se observan ciertas paradojas respecto al ideal liberal y universalista que los inspira, que ponen de manifiesto las posibles derivas excluyentes de este tipo de medidas, difícilmente compatibles con los valores de la democracia liberal que tratan de proteger. 
Palabras clave: multiculturalismo y pluralismo, ciudadanía, políticas de inmigración, integración, liberalismo.

\begin{abstract}
This paper analyzes the citizenship courses and exams recently introduced in Europe. Does the manner in which each country understands nationhood and citizenship determine the content of these programmes or are there similarities, regardless of the country's conception of nationhood? How can we explain these similarities and differences? Do these programmes reflect an idea of belonging based on a commitment to civic values that are inclusionary and universalist rather than on shared cultural traditions that are exclusionary and particularist? Or are there tensions between the normative proposal of liberal universalism and its realization in certain policies of integration?

To answer these questions, I propose an analytical framework that enables a normative evaluation of civic integration programmes in France and Germany. I, thus, carry out an in-depth analysis of the programmes, in order to explain their similarities and differences, both from a theoretical perspective and in relation to the political life in each country. Although these countries represent divergent conceptions of nationhood and citizenship, the analysis of their respective programmes reveals many similarities. However, in spite of what one might expect, both bring to light certain paradoxes regarding the liberal and universalist ideal that inspires them, which expose the possible exclusionary consequences of this type of measure, which are hard to reconcile with the liberal democratic values they are trying to protect.
\end{abstract}

Keywords: multiculturalism and pluralism, citizenship, immigration policy, integration, liberalism.

\title{
INTRODUCCION: CIUDADANÍA, POLÍTICAS DE INTEGRACIÓN Y LIBERALISMO
}

Al hilo de las directrices de la Unión Europea (Consejo de la Unión Europea, 2004), los países europeos han recurrido a distintas medidas con el objetivo de avanzar en la integración de la población inmigrante, promoviendo tanto una equiparación de derechos como un sentimiento de pertenencia que permita una mayor cohesión social. Una de esas medidas es la realización de cursos y exámenes de ciudadanía con el fin de lograr «un conocimiento básico del idioma, la historia y las instituciones de la sociedad de acogida» desde la convicción de que ese conocimiento «es esencial para que integración tenga éxito", tal y como queda recogido en el cuarto de los Principios Básicos Comunes para la política de integración de los inmigrantes acordados por el Consejo de la Unión Europea en el año 2004. Por otro lado, el mismo documento incluye una idea de integración como «un proceso bidireccional y dinámico de ajuste mutuo» (Consejo de la Unión Europea, 2004), que exige la participación, la interacción y el diálogo. Se trata, por tanto, de un modelo intercultural, que pretende superar tanto 
el monismo cultural del asimilacionismo como la imposibilidad de diálogo del multiculturalismo cerrado (Consejo de Europa, 2008).

La introducción de este tipo de programas se realiza en un momento en el que el discurso de la extrema derecha en torno a la preferencia nacional y a la necesidad de proteger nuestra identidad frente a la «amenaza» de la inmigración -sobre todo, la de origen musulmán, considerada como la máxima expresión de lo «extraño»- ha ido logrando un considerable apoyo, como se ha puesto de manifiesto en sucesivas convocatorias electorales (Innerarity y Acha, 2010). Pero al mismo tiempo, las políticas de ciudadanía en Europa están avanzando hacia un «nacionalismo cívico» (Joppke, 2007), que ha sido objeto de diferentes interpretaciones: como un proceso de convergencia en torno a modelos asimilacionistas (Joppke y Morawska, 2003) o como un giro en el que aún cabe distinguir rasgos específicos de los modelos nacionales clásicos (Jacobs y Rea, 2007). Más allá de la permanencia o no de las diferencias en cada país, sí que es posible constatar en Europa una disposición a entender la pertenencia como algo que se basa en el compromiso con valores cívicos y no tanto en tradiciones culturales compartidas. Muestra de ello es, por ejemplo, la tendencia a la liberalización de las políticas de ciudadanía por medio de la introducción de elementos del ius soli, que harían más fácil el acceso independientemente de la ascendencia (Innerarity y Acha, 2010). Sin embargo, esta apertura de las políticas de ciudadanía ha ido acompañada de la exigencia de requisitos más o menos estrictos para poder obtenerla, como asistir a cursos de integración o demostrar un nivel determinado de conocimiento del idioma y las instituciones del país de acogida por medio de un test.

Cualquier política de integración de este tipo supone una idea de quiénes somos nosotros, qué es lo que nos mantiene unidos y, por tanto, qué es lo que los potenciales nuevos ciudadanos deberían compartir para que la integración tenga éxito. Este estudio pretende analizar el contenido de los exámenes y los cursos de formación cívica en Francia y Alemania para ver qué idea de la pertenencia reflejan y, más concretamente, si en ellos tienen más peso los elementos cívicos/universalistas o los identitarios/particularistas de la ciudadanía, respondiendo de una manera u otra a la idea de nación característica de cada uno de estos dos países respectivamente.

Este trabajo parte de la tensión inherente a la institución de la ciudadanía entre inclusión universal y exclusión particularista. Por un lado, la ideología liberal que está en su base subraya el carácter esencialmente igualitario y universal del estatus de ciudadano, proporcionando un impulso hacia la inclusión de individuos y minorías en un proyecto político en torno a los derechos del individuo independientemente de su identidad peculiar. El título de ciudadano fue adoptado por la Revolución Francesa para designar la realidad simbólica de la igualdad y la trascendencia respecto a la pertenencia a grupos sociales, religiosos o culturales específicos y «considerar al individuo abstracto, sin identificación ni atributos particulares, al margen de sus determinaciones concretas» (Schnapper, 2001: 49). Pero la consolidación del Estado-nación moderno requirió del sentimiento de pertenencia nacional para asegurar su legitimación. Por eso, desde el siglo XVIII la ciudadanía se ha vinculado al Estado nacional, dentro del proceso de nacionalización de las instituciones fundamentales del Estado (Ejército, 
educación, cultura, economía). La ciudadanía es, así, un concepto que se articula a partir del reconocimiento de la igualdad individual y, al mismo tiempo, de la diferencia colectiva, que se traduce en la doble dimensión de la ciudadanía como estatus legal y como identidad.

Por eso, la ciudadanía tiene, por definición, un carácter excluyente y ha actuado de hecho como «un poderoso instrumento de cierre social» (Brubaker, 1992: 23). El conjunto de derechos civiles, políticos y sociales que se desarrollan paulatinamente a lo largo de los siglos XIX y xx hasta cristalizar en el Estado de bienestar, cuya clasificación atribuimos a Marshall, es disfrutado por aquellos ciudadanos que pertenecen a la comunidad nacional, mientras que el resto son excluidos en diversa medida. La ciudadanía es, así, «inclusiva hacia dentro» y «excluyente hacia fuera» (Brubaker, 1992: 5). El estatus de ciudadanía, aparentemente universal e inclusivo, pasa por la pertenencia a una nacionalidad, una categoría intrínsecamente particularista y excluyente. Por eso, la ciudadanía en las democracias liberales occidentales es «el equivalente moderno del privilegio feudal» (Carens, 1987: 252), ya que el disfrute de los derechos pasa por la pertenencia a una comunidad particular, permaneciendo, así, inmune a las fuerzas del liberalismo. Es la "paradoja de la modernidad política» (Wimmer, 2002): el hecho de que los principios universales de la democracia y la ciudadanía se han realizado en enclaves particularistas, excluyendo a todos aquellos que no comparten determinados caracteres étnicos o nacionales. El principio moderno de inclusión está, así, estrechamente unido a formas de exclusión étnicas y nacionales.

A partir de esta doble dimensión de la ciudadanía, universalista e incluyente, por un lado, particularista y excluyente, por otro, surgen dos tipos de cuestiones que me propongo indagar aquí. En primer lugar, si la concepción tradicional y legal de la ciudadanía y la nación en cada país determina el tipo y contenido de las políticas de integración y en concreto de los test y cursos de ciudadanía, y sí no es así, por qué. Y la segunda cuestión sería cuáles son las tensiones que la introducción de estos programas de integración cívica plantea desde un punto de vista normativo frente al ideal liberal universalista de las democracias europeas contemporáneas.

Todo parece apuntar -y esta sería la hipótesis de este trabajo- a que, en principio, cabría esperar diferencias en los programas de Francia y Alemania, dado que mantienen dos conceptos de nación diferentes, más liberal y universalista en Francia, más particularista en Alemania. Pero si no encontramos tales diferencias, ¿a qué puede deberse?

Desde estos presupuestos y para responder a las preguntas planteadas, me propongo analizar el contenido propuesto para los exámenes y cursos de ciudadanía o los contratos de integración en Alemania y Francia. Se trata de ver si en ellos se otorga un mayor peso al componente universalista -formal, cívico- o al componente particularista -identitario- de la ciudadanía. Pretendo comprobar, en definitiva, hasta qué punto reflejan un giro hacia un entendimiento postnacional o mantienen una visión identitaria, particularista de la pertenencia a una comunidad y, entonces, tendrían un carácter excluyente, difícilmente compatible con los valores de la democracia liberal. 
La selección de estos dos países se debe a que cada uno de ellos encarna uno de los modelos clásicos de nación y, en consecuencia, de integración y de ciudadanía. En Francia gira en torno a valores políticos compartidos, por lo que la integración pasa por la asimilación de dichos valores y la ciudadanía se atribuye a partir del ius soli. Alemania, en cambio, se entiende a sí misma como una comunidad étnica, basada en tradiciones comunes. De ello se deriva que no haya procurado la asimilación de los inmigrantes y que la atribución de la ciudadanía se rija por el ius sanguinis. De ahí también el interés de analizar si esas diferentes formas de entender la pertenencia se reflejan de alguna manera en el contenido propuesto para las medidas de integración cívica.

Existen trabajos en esta línea que han llevado a cabo un análisis cuantitativo, por ejemplo, del número de preguntas de carácter universalista o particularista que contienen los test en varios países de Europa (Michalowski, 2011). Esta metodología resulta interesante, pero no cabe aplicarla a los países seleccionados aquí por la distinta naturaleza de las medidas y de los materiales disponibles para quienes pretenden solicitar la ciudadanía, que van desde los modelos de posibles preguntas a los contenidos de los manuales para preparar el examen o de los cursos de formación cívica. Dada la imposibilidad de realizar un estudio de ese tipo, haré un análisis interpretativo de la documentación facilitada por cada país a las personas que pretenden acceder a la ciudadanía, desde esa doble perspectiva del universalismo y el particularismo.

En Alemania es obligatorio realizar un test y existen cursos de formación cívica para prepararlo. El currículum de los cursos de formación cívica y la batería de preguntas para el diseño del test resultan accesibles de forma gratuita en la página web del Ministerio de Inmigración (BAMF). Analizaré tanto las preguntas como el contenido propuesto para los cursos en la Directriz sobre los test y los cursos de ciudadanía ${ }^{1}$ del año 2008.

Francia no dispone de un test escrito. El conocimiento del idioma ${ }^{2}$ y de la vida en Francia es comprobado por medio de una entrevista con un funcionario del Gobierno, lo que proporciona un enorme poder de discrecionalidad al entrevistador. Dada la ausencia de un test escrito, voy a analizar dos documentos. En primer lugar, el informe Le contrat et l'integration, elaborado por el Haut Conseil a l'integration (HCI) en el año 2003, que recoge el currículum propuesto para la jornada de formación cívica a la que deberán asistir los candidatos. Y, en segundo lugar, el contenido del Livret du citoyen, publicado por el Ministerio del Interior en el año 2015 con el fin de ayudar a los aspirantes a prepararse para la conversación en la que tendrán que probar que dis-

1. Bundesamt für Migration und Flüchtlinge. 2008. Verordnung zu Einbürgerungstest und Einbürgerungskurs.

2. El requisito del conocimiento del idioma puede considerarse como algo de carácter particularista. Sin embargo, no entro a analizarlo puesto que se trata de una exigencia instrumental que no responde a un entendimiento de la pertenencia y de la integración en términos más o menos particularistas. 
ponen de un «conocimiento suficiente de la lengua, la historia, la cultura y la sociedad francesa, así como los derechos y deberes asociados a la nacionalidad», tal como lo establece el artículo 21-24 del Código Civil, tras la modificación del año 2011.

En los párrafos que siguen voy a presentar, en primer lugar, el marco analítico para evaluar normativamente los programas, definiendo qué entiendo por contenido universalista y particularista, respectivamente. A continuación, aplicaré dicho marco al análisis de los programas desarrollados en los dos países estudiados aquí. Como se apreciará, no se ven afectados de una manera clara por los conceptos clásicos de nación y ciudadanía en cada país, de forma que observamos más similitudes entre ellos de las esperadas. Por otra parte, -y es lo que trataré al final del artículo- en ambos casos encontramos tensiones entre estos programas y los ideales del universalismo liberal, que adoptan diferentes formas en uno y otro país.

\section{MARCO ANALÍTICO: CÓMO EVALUAR NORMATIVAMENTE LOS PROGRAMAS DE INTEGRACIÓN CIVICA}

El requisito de realizar cursos y exámenes de ciudadanía ha sido objeto de numerosas críticas que tienen que ver con esa doble dimensión de la ciudadanía -universalista, incluyente/particularista, excluyente- a la que me he referido. Voy a exponer, en primer lugar, los problemas que plantea la mera exigencia de realizar un test $\mathrm{o}$ asistir a un curso y, a continuación, los que se derivan de su contenido, donde explicaré qué puede considerarse como contenido universalista o particularista, respectivamente, para analizar posteriormente los casos seleccionados.

En primer lugar, no está claro que la exigencia de aprobar un test o asistir a una jornada de formación pueda considerarse una prescripción liberal, acorde con la vocación universalista de la ciudadanía, hasta el punto de que algunos autores han señalado desde una postura minimalista que «el test de ciudadanía más liberal es que no haya ninguno" (Carens, 2010: 19-20). Por un lado, este tipo de exigencias plantea el problema de que quienes son tratados como iguales cuando se trata de extraer recursos -se les exige el pago de impuestos, independientemente de cuál sea su situación-, sin embargo no lo son a la hora de imponer requerimientos para su integración. La obligatoriedad de pagar impuestos o de cumplir la ley no depende de dónde haya nacido una persona o del tiempo de residencia en el país, pero sí, en cambio, el disfrute de los derechos que garantiza la pertenencia plena y que pasa por su capacidad para acreditar unos conocimientos (Kostakopolou, 2010: 16; Carens, 2010: 19; Carrera y Guild, 2010: 31). Por eso, tienen un carácter discriminatorio que choca con el postulado de la igualdad liberal. No es lo mismo esperar que las personas adquieran de una forma espontánea o voluntaria una serie de conocimientos y competencias que les permitan desenvolverse en el país de acogida, que exigir ese conocimiento como un requisito legal para poder acceder a la ciudadanía.

Además, este tipo de medidas despiertan la sospecha de que, aunque parten del paradigma de la integración cívica, en realidad su objetivo es controlar y restringir la 
inmigración. Desde esta perspectiva, los test serían una herramienta para ayudar a los Estados a restringir la entrada de personas con baja cualificación o presuntamente no adaptables (Kostakopolou, 2010: 16; Jopkke, 2007: 5), en un contexto caracterizado por la implementación de políticas de inmigración restrictivas. De hecho, han tenido como efecto una disminución del número de naturalizaciones (van Oers, 2014).

Problemática resulta también su justificación, que es la promoción de la cohesión social (Orgad, 2010: 22). Si consideramos que el conocimiento de las instituciones, la historia y el funcionamiento de la sociedad de acogida son importantes para la estabilidad social, ¿por qué no exigirlo también -y con más razón aún- a los autóctonos, por ejemplo, al llegar a la mayoría de edad y empezar a ejercer el derecho al voto? La obligatoriedad de demostrar el conocimiento de los principios liberales presupone que solo los inmigrantes los desconocen y que solo ellos deben dar pruebas de que no es así.

Además, los test de ciudadanía podrían abrir la puerta a una extralimitación coercitiva por parte del Estado. Esto sucede cuando se pregunta no solo por el conocimiento sino también por las actitudes y los modos de vida de los ciudadanos -como se verá en el análisis del contenido propuesto para algunos exámenes-. Pero el liberalismo implica libertad para elegir ser liberal o no, mientras el modo de vida sea legal. El estado liberal no requiere que los ciudadanos crean en los principios liberales, únicamente que actúen conforme a ellos. Es perfectamente legítimo, por tanto, exigir que el comportamiento externo sea acorde con los principios del liberalismo, pero no lo es pedir la conformidad de las convicciones, que, en último término, es lo que querrían lograr dichos programas, más aún en el contexto en el que son adoptados en Europa.

A ello habría que añadir el carácter discriminatorio de algunas pruebas que se dirigen, de manera más o menos abierta, a los musulmanes. El ejemplo más claro es el de los test propuestos en el año 2005 por el estado de Baden-Württemberg, que inicialmente solo debían realizar los inmigrantes procedentes de países musulmanes, o el caso de Holanda que, en principio, lo exige a todo el mundo, pero al mirar la lista de excepciones - ciudadanos de la Unión Europea, Australia, Canadá, Japón, Nueva Zelanda, Suiza y Estados Unidos- puede apreciarse que los occidentales quedan eximidos de la obligación de realizarlo. Una medida de esta índole, que discrimina por origen, es claramente iliberal. Y, además, resulta contradictoria con una de las razones para adoptar este tipo de acciones (van Oers, 2014: 260), que es la uniformidad en los criterios para acceder a la ciudadanía, sin dejarlos al arbitrio de la autoridad correspondiente. De hecho, dado que, pese a su vocación universalista, la ciudadanía es «localmente excluyente» (Brubaker, 1992: 45), la formalización y estandarización de las diferentes medidas de integración cívica encierran un aspecto positivo, y es que quedan definidos claramente los criterios de inclusión y exclusión, mucho más cuando -como en el caso de los países estudiados aquí- se publican los materiales para prepararlos.

Más allá de las dudas que suscita la exigencia de mostrar las propias competencias cívicas, me interesa ver en qué medida el contenido de los exámenes y los cursos otorga un mayor peso al componente universalista-liberal o al particularista-nacional de la 
ciudadanía al que me he referido anteriormente. Para ello, es preciso, en primer lugar, definir qué podemos considerar, respectivamente, como contenido universalista o particularista.

Para explorar la cuestión sobre los contenidos de los cursos y exámenes de ciudadanía, resulta útil recurrir, en primer lugar, a la distinción rawlsiano-habermasiana entre el ámbito de la política y el ámbito de la ética. De acuerdo con Habermas (1996), cabe distinguir dos niveles en lo que el Estado podría exigir a los inmigrantes. El primero «consiste en la aceptación de los principios constitucionales en el interior del espacio interpretativo determinado por la autocomprensión ético-política de los ciudadanos y por la cultura política del país» (Habermas, 1996: 267). El segundo implica «una disposición a la aculturación, esto es, no solo a la aceptación externa, sino a la interiorización de los modos de vida, las prácticas y las costumbres del país de acogida» (Habermas, 1996: 267). Este segundo nivel apela a una integración de carácter ético-cultural que afecta, por tanto, a la identidad de la persona inmigrante de un modo mucho más profundo que el anterior. «El Estado democrático que tome en serio la diferenciación entre los dos niveles de integración -continúa Habermas- solo puede exigir a los inmigrantes la socialización política señalada en el primer nivel» (1996: 267). Esta manera de entender la integración no afecta a la cultura política compartida necesaria para la supervivencia de una sociedad multicultural, ya que esa identidad colectiva «está fundada en los principios constitucionales anclados en la cultura política y no en orientaciones básicas de la forma de vida cultural que predomina en un país» (1996: 267). Es la unidad social basada en el consenso de carácter procedimental sobre lo que es justo (right), no sobre lo que es bueno (good), sobre los principios institucionales, que no se derivan de una ética particular, sino que tienen un carácter exclusivamente político (Rawls, 1993: 144).

Parto, además, en este análisis de la distinción que señala Joppke (2007: 10; 2010b: 2) entre cuestiones relativas al conocimiento -ya sea de las leyes o de valores moralesy aquellas que van destinadas a comprobar la disposición interior de los candidatos, como ocurre cuando se interroga por las actitudes de las personas en relación con comportamientos concretos y se exige a los aspirantes compartir determinadas preferencias respecto a, por ejemplo, la autoridad parental o las relaciones de género.

Otro elemento útil para elaborar el marco analítico que nos permita responder a la pregunta por el universalismo y el particularismo en los programas de integración cívica, lo proporciona la distinción que hace Gray (2000) y que también utiliza Joppke (2009: 119) en su análisis de los conflictos en relación con el velo islámico, sobre las dos formas de liberalismo que conviven actualmente en Europa. Existe, por un lado, un liberalismo de corte hobbesiano, que postula la igualdad, los derechos individuales y la neutralidad, que permite la coexistencia pacífica de distintos modos de vida y «que puede emprenderse en muchos regímenes diferentes» (Gray, 2000: 12) sin que sea necesario compartir una serie de valores comunes. Este primer tipo de liberalismo es el que ha llevado a los Estados a renunciar al asimilacionismo que estaba en la base del proyecto de construcción nacional, a facilitar el acceso a la ciudadanía y a extender progresivamente los derechos, independientemente de que los individuos gozaran del 
estatus de ciudadanía o no. Pero existe también otra versión del liberalismo más ambiciosa, como "un consenso racional sobre el mejor modo de vida posible» (Gray, 2000: 11), que se manifiesta, como veremos, en determinado tipo de preguntas que interrogan a las personas sobre su adhesión a los valores liberales.

A partir de estas distinciones de carácter teórico, cabe afirmar que las preguntas relativas a los conocimientos de los candidatos no plantean, en principio, ningún problema. Sin embargo, en las que se refieren a sus valores morales, el Estado trasciende el ámbito de lo político para entrar en las convicciones que se supone que un ciudadano debería compartir. Este tipo de preguntas serían claramente incompatibles con los principios liberales -concretamente, atentarían contra la libertad de opinión y de conciencia- y quedarían muy lejos del contenido universalista de la ciudadanía, ya que, entonces, el Estado liberal estaría abierto solamente a las personas que se identifican con una serie de normas de comportamiento. Parten de un sentido «fuerte» de la pertenencia, en cuanto que se espera de los inmigrantes con un bagaje cultural diferente que compartan las normas relativas, sobre todo, a la igualdad de género o al comportamiento sexual (Michalowski, 2011: 750). El Estado puede pedir que sus ciudadanos conozcan y actúen conforme a los principios liberales, pero no que crean, que acepten dichos principios. Podría ser, entonces, considerado como liberal, por ejemplo, preguntar si varones y mujeres son iguales ante la ley (what is right), pero no cuál es la opinión del solicitante respecto a la igualdad de género (what is good) (Van Oers, 2014: 27).

Pero es posible refinar aún más este criterio de diferenciación entre conocimientos y disposiciones recurriendo también a la distinción entre el ámbito de la política y el de la ética, pero tomando éste último en un sentido diferente al que acabo de señalar, no como lo relativo a las convicciones morales del candidato sino a los valores morales en el país de acogida. Partiendo de esta distinción, también podrían considerarse como no liberales, no solo las cuestiones que se refieren a «qué es lo que el candidato considera bueno (es decir, las relativas a su disposición interior), sino también si exigen saber qué es considerado como bueno en la sociedad de acogida» (Michalowski, 2011: 752). Para el análisis que voy a realizar aquí, este tipo de cuestiones sobre la ética entraría dentro del contenido particularista, no universalista, de la ciudadanía. La razón de ello es que presuponen una homogeneidad de valores en el país de acogida, que ni existe en las sociedades occidentales ni es un presupuesto para el funcionamiento de las democracias liberales. Sí podrían ser consideradas como liberales, en cambio, las preguntas o los contenidos relativos al sistema político, la democracia y los derechos, la ley y las instituciones, es decir, las relativas al conocimiento de hechos y no de valores.

Las cuestiones relacionadas con el conocimiento de la historia del país plantean algunas dudas. En principio, no tendrían por qué entrar dentro del contenido particularista puesto que se refieren al conocimiento de hechos. Pero no todas merecen la misma consideración. Desde luego, no es lo mismo preguntar por las obras de Caspar David Friedrich que interrogar acerca de las fechas de la Segunda Guerra Mundial o la caída del Muro de Berlín. Claramente, la primera tiene un carácter particularista. Sin embargo, el segundo tipo de preguntas no serían reflejo de un particularismo cultural ya que únicamente supone un conocimiento del camino 
particular a la democracia seguido por este país concreto, un «nacionalismo constitucional» (Orgad, 2010a; 2010b) propio del Estado liberal, en el sentido de que todo Estado tiene una idiosincrasia constitucional que se refleja en su historia. Algunos autores rechazan esta perspectiva (Carrera y Guild, 2010: 31) y es cierto que no resulta fácil trazar una frontera nítida entre lo que podríamos denominar una visión sustantiva o cultural y una visión procedimental o institucional de la historia. Sin embargo, pienso que los contenidos relativos a la manera en que cada país ha entrado en la democracia pueden recibir una consideración semejante a la configuración concreta que adoptan en él las instituciones democráticas, como puede ser el sistema de gobierno o la organización territorial del Estado. Por lo tanto, los contenidos relativos a la historia constitucional del país serán considerados universalistas en este análisis.

Finalmente, sí que entrarían dentro del contenido particularista aquellos aspectos que se refieren a las costumbres, la cultura y las tradiciones del país ${ }^{3}$.

En conclusión, para el análisis realizado aquí, el contenido de un test o curso de formación cívica será universalista si se refiere únicamente a cuestiones relativas al conocimiento de las leyes, los derechos y deberes o las instituciones y su historia. Será particularista, en cambio, si incluye aspectos relativos a las actitudes de los candidatos o al conocimiento de los valores morales, las costumbres y tradiciones culturales de la sociedad de acogida. El siguiente cuadro presenta de forma esquemática esta clasificación.

TABLA 1.

CONTENIDO PARTICULARISTA/UNIVERSALISTA EN LOS CURSOS Y EXÁMENES DE CIUDADANÍA

\begin{tabular}{llll}
\hline \multicolumn{2}{c}{ Universalismo } & \multicolumn{1}{c}{ Particularismo } \\
\hline Conocimiento & Derechos y deberes & Actitudes & \\
\cline { 2 - 4 } & Leyes, instituciones & Conocimiento & Valores morales \\
\cline { 2 - 3 } & Historia de las instituciones & & Costumbres y tradiciones \\
\hline
\end{tabular}

Fuente: elaboración propia.

Una vez establecido el marco analítico, pasaré a aplicarlo al análisis de los programas desarrollados por los dos países seleccionados.

3. Quisiera señalar otra cuestión interesante, aunque no entra dentro del objeto de este trabajo, por la selección de países realizada. Es la práctica llevada a cabo en Holanda, consistente en mostrar a los candidatos videos de mujeres en top less o de una pareja homosexual. Por un lado, mostrar los videos no sería iliberal puesto que únicamente se refiere al conocimiento de un hecho y es que determinados comportamientos son legales en el país de acogida. Sin embargo, de forma indirecta se está preguntando también por la tolerancia hacia esos comportamientos, lo cual es ya una actitud, totalmente necesaria, por otra parte, para la vida en una sociedad liberal. 


\section{ANALISIS DEL CONTENIDO DE LOS PROGRAMAS Y SU ORIENTACIÓN LIBERAL Y UNIVERSALISTA}

\section{Los exámenes de integración en Alemania}

Alemania ha optado tradicionalmente por el derecho de sangre, la transmisión de la nacionalidad por descendencia. Sin embargo, a partir de finales de los noventa empieza a introducir una serie de reformas en la línea de las emprendidas por otros países europeos, que tienden a flexibilizar los requisitos para el acceso con un doble objetivo: facilitar la naturalización e impulsar el conocimiento del alemán y del Estado de derecho. Todo ello como un medio para lograr la integración de las segundas generaciones de inmigrantes en la sociedad alemana, entendida como algo que responde al interés general. En primer lugar, la reforma de la Ley de Nacionalidad (Staatsangehörigkeitsgesetz $)^{4}$, llevada a cabo en el año 2000 por el Gobierno de coalición de los socialistas y los verdes, modifica sustancialmente la normativa vigente desde 1913, introduciendo elementos del derecho de suelo, aunque sujetos a condiciones más estrictas que en otros países europeos (Innerarity y Acha, 2010). La nacionalidad resulta, en principio, más accesible, pero al mismo tiempo se hace más difícil obtenerla, ya que la ley introduce una serie de restricciones destinadas a probar la integración de las personas solicitantes, como mostrar su competencia oral y escrita en alemán y su disposición a respetar la Constitución y el orden político democrático5.

Un segundo cambio importante se produjo con la entrada en vigor, el 1 de enero de 2005, de la Ley Federal de Inmigración ${ }^{6}$ y de la Ley sobre el Permiso de Residencia ${ }^{7}$. En primer lugar, el requisito del conocimiento de la lengua va a ser necesario, no solo para la ciudadanía, sino también para obtener el permiso de residencia permanente. Además, se incluye en ambos casos la obligación de realizar cursos de integración para el conocimiento tanto del idioma como de la historia y el sistema político alemán ${ }^{8}$. Finalmente, la ley establece como requisito para la adquisición de la nacionalidad

4. La ley no utiliza el término Staatsbürgerschaft (ciudadanía), que tiene un sentido político-jurídico, sino Staatsangehörigkeit (nacionalidad). Por ello empleo aquí el término «nacionalidad», como lo hace la ley alemana.

5. Pese a las presiones de la CDU/CSU, que consideraba que estas restricciones no probaban suficientemente la integración, finalmente no se introdujo la exigencia de mostrar un conocimiento del sistema político del país.

6. Gesetz zur Steuerung und Begrenzung der Zuwanderung und zur Regelung des Aufenthalts und der Integration von Unionsbügern und Ausländern. Vom 30.Juli.2004.

7. Gesetz über den Aufenthalt, die Erwerbstätigkeit und die Integration von Ausländern im Bundesgebiet. Vom 30.Juli.2004.

8. Ya desde los años noventa, Alemania había empezado a ofrecer este tipo de cursos a los expatriados de Europa del Este y Rusia que querían recuperar la nacionalidad alemana, con el fin de que pudieran prepararse para la realización de un test que debían pasar antes de poder entrar legalmente en el país. Una vez allí, recibían cursos de orientación cívica y del idioma durante 
aprobar previamente un test de integración y demostrar un nivel de conocimiento del alemán correspondiente a un B1. Esta ley es elaborada en un contexto marcado no solo por los atentados del 11-S, sino también por los estallidos de violencia en los barrios de población inmigrante (Innerarity, 2007) o los crímenes de honor en algunas familias procedentes de Turquía. Por eso, señala como un impedimento para obtener la nacionalidad la existencia de indicios que pudieran mostrar un compromiso por parte del solicitante con actividades contrarias a la Constitución o el apoyo a organizaciones terroristas (EUDO, 2015: 18) .

En este contexto, el conflicto más significativo se produce en el año 2005 en el estado de Baden-Württemberg. Aunque las cuestiones relativas a la nacionalidad son competencia de la Federación, el Gobierno conservador de este Land aprueba una regulación administrativa para orientar a la autoridad encargada de probar la disposición del candidato a respetar el orden político democrático ${ }^{10}$. Contiene una serie de preguntas que no se refieren al conocimiento de las instituciones de la sociedad alemana sino a las actitudes y opiniones de los candidatos respeto a una serie de temas, una gran parte de ellos relacionados con la igualdad de género -los matrimonios forzosos, la manera de vestir, la poligamia, la autoridad de los padres- así como sobre el apoyo a actividades terroristas, que terminaba con una declaración de lealtad a la Constitución. Se pregunta, por ejemplo: «¿Considera aceptable que un hombre encierre en casa a su mujer o su hija para evitar que le provoque 'vergüenza pública’?», «¿Está usted de acuerdo con que la mujer debe obedecer a su marido y que este puede pegarle cuando no le obedece?» (Staatsministerium Baden-Württemberg, 2005). La autoridad debía interrogar al candidato para comprobar su compromiso con los valores de la democracia, hasta el punto de que cabía denegar la ciudadanía si existía alguna duda sobre sus "convicciones internas» (Wolfrum y Röben, 2006).

El estado de Hesse propone también en el año 2006 una lista de cien preguntas para elaborar el test de ciudadanía en esta misma línea. Incluye cuestiones como, por ejemplo: «Una mujer no debería mostrarse en público ni viajar sin la compañía de un miembro masculino de su familia: ¿̨cuál es su opinión?». Además de este tipo de temas,

seis meses. Lo que se hace ahora es extender al resto de los inmigrantes la política relativa a los expatriados y a los inmigrantes étnicos.

9. Un tema objeto de crítica recurrente en la política de nacionalidad es el hecho de que la adquisición de la nacionalidad alemana se reduce al cumplimiento de una serie de requisitos meramente formales, lo que no contribuye a mostrar el valor que tiene en sí misma. Sin embargo, no fue posible llegar a un acuerdo sobre la introducción de un juramento de lealtad, como el que existe, por ejemplo, en Estados Unidos. Únicamente se pide declarar -en el momento de recibir el certificado de nacionalidad- la voluntad de cumplir la Constitución y las leyes vigentes en la República Federal: "Ich erkläre feierlich, dass ich das Grundgesetz und die Gesetze der Bundesrepublik Deutschland achten und alles unterlassen werde, was ihr schaden könnte" (Staatsangehörigkeitsgesetz, $₫ 16$ ).

10. Staatsministerium Baden-Württemberg, 2005. Gesprächsleitfaden für die Einbürgerungsbehörden. 1.09.2005. 
interroga también por aspectos relativos a la cultura, como, por ejemplo, el motivo de un cuadro o el nombre de filósofos alemanes.

Estos exámenes generaron un fuerte rechazo en la opinión pública -que se refiere a ellos como "test de conciencia» (Gessinnungstest) (Ataman, 2008; Die Zeit, 2010)- al ser considerados como una injerencia ilegítima por parte del Estado en el ámbito de las convicciones personales y que, en palabras del diputado de Los Verdes, Joseph Winkler, "sitúa a los musulmanes bajo una sospecha escandalosa» (Reimann, 2006). De hecho, la realización de lo que muy pronto se denominó «Muslim Test» (Speckner, 2006; Reimann, 2006; Deutschewelle, 2006) estaba prevista inicialmente solo para los candidatos procedentes de países pertenecientes a la Organización de la Cooperación Islámica. Sin embargo, tras constatar el carácter discriminatorio de esta medida, se aplica siempre que existan dudas sobre la aceptación de los valores en los que se basa el orden político alemán, independientemente del lugar del que provengan los candidatos. Para evitar este tipo de problemas, el Gobierno ha llevado a cabo una relativa estandarización de su contenido, que puede considerarse ya en sí misma un avance en la universalización de los test, puesto que el acceso no va a depender de la subjetividad de una autoridad que deba valorar la capacidad de integración del interesado. Concretamente, en el año 2008, el Ministerio del Interior publica una directiva $^{11}$ que establece el contenido de los cursos previos a la obtención de la ciudadanía. Consta de tres unidades - $V$ Vida en democracia», "Historia y responsabilidad», "Persona y sociedad» (Leben in der Demokratie, Geschichte und Verantwortung, Mensch und Gesellschaft)-, de las cuales la más amplia es la que se refiere a la «Vida en democracia». Esta incluye una serie de conocimientos básicos sobre la Constitución, los derechos fundamentales, el Estado de derecho, el Estado de bienestar, la igualdad entre hombres y mujeres, los símbolos del Estado, la resolución de conflictos en una sociedad democrática, los derechos y obligaciones o la participación política.

El módulo dedicado a "Historia y responsabilidad», se refiere a conocimientos básicos sobre la historia política reciente, como algo necesario para comprender la actualidad de Alemania, ese «nacionalismo constitucional» (Orgad, 2010a) al que antes me he referido.

Finalmente, el módulo «Persona y sociedad» afronta una batería de cuestiones relacionadas con la vida social en Alemania, que posibilitan el ejercicio concreto de los derechos. Hace una referencia especial al cambio cultural como consecuencia de las migraciones internacionales y del establecimiento de minorías étnicas en el país. Aspectos fundamentales en esta línea son el pluralismo religioso, el lugar de la religión en la sociedad, la separación Iglesia-Estado y las relaciones interculturales.

Además de las orientaciones para la realización de los cursos, la directiva contiene un catálogo de trescientas preguntas de carácter general y diez específicas de cada Land, de las que hay que seleccionar treinta y tres para confeccionar el examen, tres de

11. Bundesministerium des Innern 2008. Verordnung zu Einbürgerungstest und Einbürgerungskurs. Vom 5.August. 2008. 
ellas relativas al Land en el que vive la persona solicitante. Esta debe responder correctamente en sesenta minutos al menos a diecisiete de ellas.

El contenido tanto del programa de formación como de las preguntas es mayoritariamente universalista desde los criterios de análisis utilizados en este estudio. En primer lugar, hay que señalar que únicamente se refieren a cuestiones cognitivas, renunciando a cualquier intento de verificar las actitudes de los candidatos que encontrábamos en los test del estado de Baden-Württemberg.

Además, la mayor parte de los temas se refieren a la política (qué significa que Alemania es un Estado de derecho, cuándo puede limitarse la libertad de expresión, cómo se llama el presidente de la República), la historia reciente (quién construyó el Muro de Berlín, cuándo tuvo lugar la Segunda Guerra Mundial) o la geografía de Alemania (qué países limitan con Alemania) y solamente algunas aluden a cuestiones que puedan tener un contenido moral (por ejemplo, el matrimonio, la prohibición de la poligamia, la tolerancia de la homosexualidad, la posibilidad de contraer matrimonio con alguien de otra confesión religiosa, a quién corresponde la responsabilidad de la educación de los hijos, la igualdad entre hombres y mujeres). Sin embargo, en ningún caso se pregunta por las actitudes respecto a ese tipo de prácticas, ni por su consideración moral en el país de acogida, sino únicamente por el conocimiento de lo que está legalmente permitido en Alemania.

También se omiten las preguntas relativas a temas culturales y que sí aparecían en versiones anteriores aplicadas en algunos de los Länder, -el caso de Hesse- como, por ejemplo, el autor de determinado cuadro o el compositor de una pieza musical. Si hay cuestiones relativas a la cultura en esta versión final estandarizada, estas se refieren únicamente a lo que es la vida cotidiana en Alemania -verschiedene Aspekte des gesellschaftlichen Zusammenlebens in Deutschland (Verordnung, 2008)-, la cultura en la medida en que afecta a la vida del solicitante, teniendo en cuenta, de manera especial, la pluralidad cultural del país, en parte, como consecuencia de la inmigración y la manera de resolver los conflictos que en este campo puedan plantearse ${ }^{12}$.

Puede resultar sorprendente, en principio, el contenido universalista del test en Alemania, un país caracterizado por un modelo de ciudadanía bastante cerrado, que hasta hace bien poco ha sido accesible solamente a aquellas personas que pudieran demostrar antecedentes alemanes. Es una muestra de que Alemania se entiende a sí misma de una forma bastante más abierta que lo que presuponen quienes apelan a la Leitkultur ${ }^{13}$, como algo opuesto a la pluralidad cultural.

12. Se pregunta, por ejemplo: «¿En qué fiesta es costumbre disfrazarse en Alemania?» o «¿De dónde procede la mayor parte de la inmigración en Alemania?» o "¿Cómo se denominaba a los inmigrantes que llegaron a Alemania en los años sesenta?».

13. El término Leitkultur, introducido por el sociólogo Bassam Tibi en los años noventa, alude a un concepto controvertido que aparece de forma reiterada desde el año 2000 en los debates sobre la identidad nacional, asociado a una visión monista de la cultura alemana que los inmigrantes deberán asimilar, como algo contrapuesto al multiculturalismo (Leitkultur statt Multikultur). 
Pueden encontrarse diversas explicaciones a este hecho. En primer lugar, quizá este planteamiento más abierto, más universalista de los cursos y exámenes de ciudadanía sea, en parte, una reacción a la polémica surgida a partir de la introducción de los test de conciencia en el estado de Baden-Württemberg. Es cierto que también en el examen actual a nivel federal se pregunta por el conocimiento de cuestiones relativas, por ejemplo, a la igualdad de género, pero sin interrogar acerca de las disposiciones internas de los candidatos. La crítica que ese tipo de test provocó en la opinión pública alemana, al ser considerado como una medida claramente discriminatoria dirigida contra la población musulmana, hizo que el Ministerio de Inmigración rechazara explícitamente esa clase de cuestiones.

Pero la paradoja se resuelve desde una mirada más amplia a lo que ha sido el modelo de integración y de gestión de la diversidad cultural adoptado en Alemania desde la Segunda Guerra Mundial y no solo por una razón meramente coyuntural, como puede ser el rechazo provocado por los Muslim-Test. Alemania ha renunciado desde el primer momento a adoptar medidas asimilacionistas por varias razones. En primer lugar, porque su aplicación podría llevar a los países de su entorno a rememorar las políticas desarrolladas por el Tercer Reich. Pero, además, Alemania ha considerado hasta hace bien poco que la inmigración es algo que está de paso, por lo tanto, no solo no tiene mucho sentido tratar de que los inmigrantes asimilen la cultura del país, sino que, además, dejar que los Gastarbeiter reproduzcan sus prácticas culturales es algo que, al tiempo que favorece la paz social mientras están aquí, facilitará que vuelvan a su país de origen una vez que han desempeñado la función para la que vinieron (Innerarity, 2012). Las modificaciones introducidas en las políticas de nacionalidad alemana y, más concretamente, la adopción de este tipo de programas cívicos, muestran, por tanto, un cambio en la percepción de la ciudadanía, que ahora empieza a considerarse como algo de interés público, resultado de una modificación en la manera de entender la inmigración, que deja de ser vista como algo de carácter temporal, abandonando el tópico de que "Alemania no es un país de inmigración», presente en el debate público desde los años setenta. La introducción de los cursos y exámenes puede verse, así, como una institucionalización tanto de la inmigración como del proceso de naturalización. Sin embargo, este cambio de mentalidad no se produce de forma homogénea en todos los partidos políticos ni en el conjunto de la sociedad. Si para el SPD y sus socios en el momento de las reformas, la adquisición de la nacionalidad es un requisito para la integración, la coalición CDU/CSU argumenta que la naturalización sería el punto de llegada -algo así como un premio- del proceso de integración. Estas diferencias se manifiestan también en la actitud hacia la doble nacionalidad. Mientras que liberales y socialdemócratas son partidarios de ampliar los casos en los que cabe permitirla, como una muestra de lealtad a dos naciones, la CDU/CSU, en cambio, desde una concepción más etnocultural de la ciudadanía, lo considera, precisamente, una señal de falta de integración (Bertossi y Hajjat, 2013: 21).

Del análisis del caso alemán podemos concluir con Michalowski (2010) que el modelo de ciudadanía no determina cuál vaya a ser el contenido de los exámenes y cursos, ya que en un país con una concepción étnica de la ciudadanía y en el que, 
por tanto, cabría esperar encontrar una orientación particularista, las cuestiones propuestas son de carácter claramente universalista. Como hemos visto, la mayor parte de los temas se refieren al conocimiento de las instituciones, los derechos, la historia reciente o los símbolos del país, pero se abstienen de incluir cuestiones relativas tanto a un nacionalismo cultural como a la moral o a las disposiciones interiores de las personas.

\section{Los contratos de acogida e integración en Francia}

La implantación en Francia de los llamados «contratos de acogida e integración» $(\mathrm{CAI})^{14}$ en el año 2003 se produce en un contexto en el que la cuestión de la ciudadanía republicana experimenta una profunda politización en el marco del debate público en torno a la identidad nacional y la inmigración. Esto es debido, entre otros factores, al éxito del Frente Nacional en las elecciones presidenciales del 2002, los debates sobre la amenaza que para los valores de la República supuestamente plantean algunos grupos religiosos y que conducen a la prohibición de los símbolos ostensibles en el año 2004 (Innerarity, 2012) o las revueltas en los barrios de inmigrantes desde los años ochenta, que encontrarán su punto álgido a finales del año 2005 (Innerarity, 2009). Por eso, a diferencia de lo sucedido en las décadas anteriores, esta repolitización de la ciudadanía no se va a centrar en la cuestión del acceso, sino en el reto que supone la diferencia cultural y religiosa para la identidad nacional (Bertossi y Hajjat, 2013). Se trata de un "conflicto entre dos identidades» (Innerarity, 2007), entre el islam y los valores de la República y, de manera especial, la laicidad y la igualdad de género. El «problema de la integración» (HCI, 2006) va a ser entendido, por tanto, como algo directamente relacionado con la cuestión de la identidad francesa. Prueba de ello será la creación del Ministerio de Inmigración e Identidad Nacional por parte del Gobierno de Sarkozy en el año 2007.

En estas circunstancias, el Gobierno conservador de Raffarin introduce en 2003 los CAI, por los que el candidato se compromete a respetar los derechos y deberes de la ciudadanía. Adoptados inicialmente por algunos departamentos como algo de carácter voluntario, la Ley de Inmigración e Integración de $2006^{15}$ establece su obligatoriedad para todo el país a partir de 2007, tanto para obtener un permiso de residencia de larga duración (a partir de tres meses), como para acceder a la nacionalidad. El cumplimiento del contrato condiciona la concesión o renovación del permiso de residencia y, en último término, el acceso a la ciudadanía por naturalización. Finalmente, los derechos y deberes del año 2003 se convierten en «los principios y valores fundamentales de la República» en la Ley sobre Inmigración, Integración y Nacionalidad,

14. Haut Conseil a l'integration. 2003. Le contrat et l'integration.

15. Assemblée Nationale. 2006. Loi n. 2006-911 du 24 juillet 2006 relative à l'immigration et à l'intégration. 
del año $2011^{16}$, que introduce también el conocimiento de «la historia y la cultura francesa», que deberá ser probado a lo largo de una entrevista antes de firmar el CAI.

El contrato consta de dos partes: una general, que incluye el compromiso de respetar las leyes y valores de la República y de asistir a una jornada de formación cívica para los recién llegados y, en caso de que sea necesario, una formación suplementaria en el idioma y en el conocimiento de la vida social en Francia, es decir, se les exige mostrar su voluntad de integrarse. Por su parte, el Estado se compromete a proporcionar gratuitamente la formación cívica y lingüística que el inmigrante deberá seguir.

A diferencia de otros países, como Alemania, Gran Bretaña o España, aquí se trata de una medida destinada a "ayudar a la integración en la sociedad francesa» (OFII, 2015). Es decir, la integración -el compromiso de integrarse- no es, en este caso, un requisito para obtener la ciudadanía, sino algo que se exige incluso antes de que ese proceso de integración comience. Esto se explica por el hecho de que la lectura cultural del «fracaso de la integración», que afecta de manera especial a la población procedente de las antiguas colonias, va a suponer un giro en la política francesa de integración que ahora va a promover lo que en el debate público se denominó una «inmigración escogida» con el fin de lograr una «integración exitosa» (Bertossi y Hajjat, 2013). De ahí el énfasis en la selección de inmigrantes que muestren de antemano su compatibilidad con la sociedad francesa. Este hecho permite afirmar, ya antes de entrar a analizar el contenido de los cursos, que los CAI - por paradójico que pueda resultar, dada la idea de nación típicamente francesa- van a ser más restrictivos que los exámenes cívicos de Alemania.

Hay otro aspecto peculiar de Francia y es que -como he señalado- no se trata de un examen, sino de un contrato, que tiene por objeto «refundar la política de integración» (HCI, 2003: 4), entendida como «la definición y realización de un proyecto compartido» (HCI, 2003: 83), por medio de una acción positiva, un contrato entre el Estado y quienes viven en el territorio. Ya la utilización del término «contrato» evoca el mito fundacional de la República (Beaujeu, 2008: 32): «Se trata de un contrato republicano, inscrito en una lógica de derechos y obligaciones recíprocas entre el Estado y el individuo» (HCI, 2003: 84), una reformulación del pacto, al que ahora acceden los inmigrantes. De ahí también la importancia concedida a la formación cívica, que no encontramos en otros países, y que aquí es, incluso, objeto de un compromiso formal entre el Estado y el inmigrante. Se trata de «una acción contractual y de una responsabilidad compartida, por la que el Estado se compromete a asegurar la formación lingüística, cívica, social y profesional, a la vez que los contratantes se comprometen a respetar las leyes de la República» (HCI, 2003: 119). El informe subraya que se trata de "una acción individualizada, atenta a los derechos de las personas y no solamente a los grupos étnicos» (HCI, 2003: 119). Se expresa aquí, por tanto, algo que es común a todos los programas de integración cívica y que consiste en la idea de que el

16. Assemblée Nationale. 2011. Loi. 2011-672 du 16 juin 2011 relative à l'immigration, à l'intégration et à la nationalité. 
proceso de integración es algo de carácter individual, algo que depende de la acción del individuo, $\mathrm{y}$, al mismo tiempo, un proceso bidireccional.

¿Cuál es el contenido de los cursos de formación cívica? El informe del HCI Le contrat et l'integration, del año 2003, contiene las directrices fundamentales al respecto. Un primer aspecto destacable es el hecho de que a lo largo de todo el informe encontramos reiteradas referencias expresas a la intención de evitar la «lógica asimilacionista", la identificación de la integración con la asimilación. "No se puede exigir al inmigrante una integración ética, es decir, renunciar a sus orígenes. Solo se debe exigir una integración política» (HCI, 2003: 4). A diferencia de la asimilación, «la integración es universalista, en cuanto que permite a cada uno permanecer él mismo» (HCI, 2003: 111). Lejos de toda voluntad de asimilación de los comportamientos, la integración «se refiere y debe referirse solamente a la ley común, es decir, a la Constitución» (HCI, 2003: 85). En este sentido, el informe alude expresamente a una «integración cívica», que trasciende "las identidades familiares, étnicas o culturales» (HCI, 2003: 106), de forma que «ciudadanía e identidad pertenecen a dos registros diferentes» (HCI, 2003: 106). «La integración cívica debe respetar la identidad de cada persona y desmarcarse de la asimilación» (HCI, 2003: 106). No se trata de «imponer nuestras costumbres», sino de "hacer respetar nuestras leyes» (HCI, 2003: 4). Por eso es importante darlas a conocer de la manera más clara y sencilla posible. Este énfasis en la integración cívica se halla en clara consonancia con la "filosofía» (Favell, 1998) francesa de integración republicana, que pasa por la adopción de los principios liberales, universalistas.

Llama la atención esta insistencia en el carácter cívico de la integración y, sobre todo, en la renuncia a la asimilación, cuando, como hemos visto, el "problema de la integración» es percibido como una cuestión de valores y que afecta, fundamentalmente, a las personas procedentes de los territorios coloniales, cuyas costumbres son consideradas como contrarias a los principios de la República. De hecho, el informe del HCI contiene reiteradas alusiones a la necesidad de que los cursos insistan en todo lo relacionado con la igualdad entre hombres y mujeres, la igualdad en el ejercicio de la autoridad parental o la integridad física, e incluye un amplio anexo sobre la mutilación genital y sobre los matrimonios forzados. Son cuestiones que, aunque, efectivamente, no sean prácticas exclusivas de los musulmanes, ni todos ellos las acepten, están presentes en el imaginario colectivo como algo que supone, no solo un obstáculo para su integración sino, incluso, una amenaza para los valores republicanos. Por este motivo resulta pertinente analizar el contenido propuesto para las jornadas de formación cívica y ver hasta qué punto se corresponde con ese rechazo expreso de cualquier pretensión asimilacionista, apostando realmente por el universalismo propio de un modelo republicano.

De acuerdo con el informe del HCI del año 2003, los cursos de formación cívica incluyen una presentación de los principios y valores fundamentales de la República, los derechos y deberes de los ciudadanos. Concretamente, los cursos deben cubrir los siguientes cinco aspectos: 
a) En primer lugar, las leyes comunes a la República. Se trata de explicar qué significa que la república francesa es democrática, laica, social y descentralizada, así como el sentido del lema («libertad, igualdad, fraternidad») y de sus símbolos (presidente de la República, bandera, Marianne, himno).

b) En segundo lugar, los derechos del hombre y del ciudadano y los derechos sociales. El informe va desgranando el significado de las principales libertades y de la igualdad, subrayando la necesidad de prestar una atención particular a la igualdad entre hombres y mujeres y a la laicidad. Así, hace una referencia expresa a la prohibición de la poligamia, de los matrimonios forzados y a la protección por ley de la integridad corporal. Alude también a la historia de la conquista de la igualdad entre hombres y mujeres (el trabajo y el voto de las mujeres, la autoridad parental), que podría incluirse dentro de lo que hemos denominado «historia constitucional». Este mismo apartado recoge la explicación del sentido de la fraternidad y la solidaridad, que se expresa mediante los servicios públicos. La contrapartida de esta solidaridad es la responsabilidad individual, sobre todo a la hora de pagar los impuestos y las cotizaciones sociales, que tienen por objeto tanto la financiación de esos servicios como la redistribución en beneficio de la colectividad y de ahí la importancia de no eludirlos. El sistema de protección social traduce el esfuerzo de todos en beneficio para los enfermos, la familia, los ancianos o los discapacitados. Este sistema compromete la responsabilidad de todos. Aunque la solidaridad se manifiesta también a través de la vida asociativa y la familia, subraya aquí la necesidad de evitar el repliegue comunitario.

c) El tercer apartado se refiere a las instituciones de la República: La organización política, los poderes ejecutivo, legislativo y judicial, los poderes locales, su carácter democrático, el sufragio universal.

d) El cuarto capítulo contiene todo lo relativo a la ciudadanía y el acceso a la nacionalidad: qué es la ciudadanía, consecuencias jurídicas de los diferentes estatus, modos de atribución, referencia al compromiso personal con la nación.

e) La jornada cívica incluye también una formación específica para las familias, en la que se informa sobre la obligatoriedad de la escuela de los 6 a los 16 años, los deberes de la autoridad parental, los derechos de la infancia y -una vez massobre la igualdad entre hombres y mujeres.

Finalmente, el informe señala que la conclusión de la jornada «debe evocar la cultura y la historia, el enriquecimiento de la cultura francesa por las aportaciones de otras culturas, así como los aspectos económicos y sociales de la vida en Francia» (HCI, 2003: 95).

Más allá de esto, la única referencia a cuestiones identitarias apunta a la necesidad de conocer la lengua francesa, pero como algo de carácter instrumental y no como un rasgo que define la identidad del país. Además, apunta también que «aunque no se le pueda exigir que renuncie a sus costumbres cuando no contravengan las leyes de la República», sí que es necesario que el inmigrante "conozca la historia del país en el que 
va a vivir» (HCI, 2003: 113). Pero solo se exige al extranjero conocer las tradiciones, en ningún momento habla de aceptarlas o compartirlas.

En esta misma línea y en consonancia con su insistencia en la integración cívica y el universalismo, el informe alerta expresamente del peligro que comporta no distinguir adecuadamente entre el ámbito del derecho, las costumbres y la moral, lo que conduciría a presentar las leyes y principios de la República como si fueran meros "productos de un tradición histórica que debe respetarse por su antigüedad» (HCI, 2003: 84), enseñar las leyes como si fueran «valores de una catequesis que únicamente requieren creencia y obediencia» (HCI, 2003: 84), evocar la Constitución nacional "como si fuera algo propio de una comunidad particular y contingente» (HCI, 2003: 84). Los valores y principios de la República, como los derechos del hombre y del ciudadano, trascienden la particularidad de la nación francesa, subraya repetidas veces el informe.

Desde los criterios de análisis propuestos en este estudio, los CAI tienen un carácter mayoritariamente universalista por diversos motivos. En primer lugar, solo encontramos referencias a cuestiones cognitivas, sin entrar en temas que tengan que ver con las disposiciones interiores de los individuos. Es cierto que exigen el compromiso de respetar los principios y valores de la República, pero en ningún momento exige compartirlos.

En segundo lugar, por lo que se refiere al tipo de conocimientos requeridos, solamente alude a cuestiones que tienen que ver con el campo de la política (las instituciones, los símbolos) y, sí, los cursos incluyen también formación en valores, pero se trata siempre de valores políticos (igualdad, solidaridad). Se refieren, por tanto -como reiteradamente manifiesta el informe del HCI del año 2003- solamente al ámbito de lo político y no de la moral. Finalmente, es cierto que aparecen referencias a la historia, pero hasta aquí se trata de la historia de los principios y valores de la República, que entrarían dentro de esa "historia constitucional» anteriormente reseñada.

Sin embargo, como he señalado antes, al conocimiento de «los derechos y deberes de la ciudadanía», se va a añadir también en el año 2011 «la historia, la cultura y la sociedad francesa». Tras ese cambio, el Ministerio del Interior ha editado recientemente un Livret du citoyen (2015) con el objetivo de ayudar en la preparación de la prueba. En él, los contenidos propios de la jornada de formación cívica se van a complementar ahora con un capítulo sobre los principales hitos de la historia de Francia desde la prehistoria (cuevas de Lascaux), la romanización, algunos personajes de la Edad Media, la Edad Moderna, la Revolución Francesa, los siglos XIX y XX (desde san Luis hasta Juana de Arco, Enrique IV, los ilustrados, Luis XIV, Napoleón o Víctor Hugo), los principales acontecimientos del siglo Xx (las dos guerras mundiales, la descolonización, mayo del 68, el Tratado de Roma, la instauración de la mayoría de edad a los dieciocho años o la abolición de la pena de muerte). Incluye también un capítulo sobre cultura, en el que presenta a personajes históricos de origen extranjero (Guillaume Apolinaire, Josephine Baker, Marc Chagall, Marie Curie, Vassily Kandinsky, Georges Moustaki). Cuestiones todas ellas propias de un nacionalismo cultural y que reflejan, por tanto, un aumento del contenido particularista respecto a las recomenda- 
ciones del año 2003. Sin embargo, a pesar de la introducción de elementos de carácter cultural particularista, en conjunto, el peso de las cuestiones que caen del lado del universalismo continúa siendo considerablemente mayor.

\section{CONCLUSION: TENSIONES Y PARADOJAS DE LOS PROGRAMAS DE INTEGRACIÓN}

Una primera conclusión que podemos extraer de los casos analizados es que la idea de nación y de ciudadanía de un país no tiene por qué determinar el contenido de los programas de integración cívica. Como hemos visto, pueden ser tan universalistas en Alemania como en un país con un régimen de ciudadanía más abierto, como es Francia. Es más, en los dos casos estudiados encontramos justamente lo contrario: los CAI de Francia, en su versión más reciente, tienen un contenido más particularista que los exámenes y las jornadas de formación en Alemania. Tal como he señalado, el material para prepararse no se limita a cuestiones políticas, sino que abarca también conocimientos sobre la cultura y la historia de Francia. Es cierto que también en Alemania los cursos contienen referencias a acontecimientos históricos, pero en el caso de Francia, no solo incluyen cuestiones que hemos denominado de nacionalismo constitucional, sino también bastantes aspectos culturales, a la vez que hacen un repaso de los principales acontecimientos históricos del país, remontándose, incluso, hasta la prehistoria.

$\mathrm{Al}$ margen de las diferencias entre los países, la mayor parte del contenido de los programas de integración cívica tanto en Francia como en Alemania tienen un carácter universalista. Por ello, de acuerdo con Joppke (2007: 16), si bien los países europeos estarían utilizando un medio no liberal -pedir evidencias a algunos futuros ciudadanos sobre su conocimiento del país- para lograr un objetivo liberal -que los candidatos adquieran las competencias para desenvolverse con éxito en la sociedad de acogida- las cuestiones a las que se refieren no tienen un carácter particularista sino que, en su gran mayoría, atañen al funcionamiento del Estado o a la vida cotidiana en una sociedad liberal, más allá de los particularismos nacionales.

Sin embargo, el tipo de políticas de integración que analizamos aquí revelan un liberalismo fuerte, en el sentido apuntado por Gray (2001) al que antes me he referido, que se manifiesta abiertamente en el caso de las preguntas dirigidas a comprobar la adhesión de las personas a los valores liberales -que, como hemos visto, se han ido rechazando progresivamente-, pero también de una manera más sutil al exigir un conocimiento de las normas de funcionamiento de la sociedad de acogida que, desde luego, no se aplica a los autóctonos.

Es un hecho que ha sido la presunta deslealtad de los inmigrantes musulmanes y la incompatibilidad de su visión del mundo con los valores del liberalismo lo que ha dado lugar a la aparición de los programas de integración cívica en Europa. Y esa confrontación con el islam ha reforzado la versión sustantiva del liberalismo. Por eso, junto al aprendizaje de la lengua se exige también el conocimiento de principios y 
valores de la democracia liberal considerados como potencialmente universales. Pero ese universalismo puede convertirse, de hecho, en una ideología particularista y excluyente. Es lo que ocurre, por ejemplo, con la laicidad en Francia, la versión local del principio liberal de la neutralidad del Estado, con el que no casan las prácticas de determinados grupos. Como hemos visto, este es un aspecto fundamental del programa de formación cívica. La laicidad, como un proyecto político en torno a los derechos del individuo al margen de su identidad particular es un rasgo que define la identidad francesa. Así lo recoge la Constitución de $1958^{17}$ y, más recientemente, el informe de la Comisión Stasi señalaba que la laicidad "es un elemento del pacto republicano» (Comisión Stasi, 2003: 1.2.2), "forma parte de nuestra historia colectiva» (Comisión Stasi, 2003: 1.1). Y esta traducción francesa de la neutralidad es lo que justifica, por ejemplo, la prohibición del velo en el año 2004, como algo que no es compatible con ese aspecto de la identidad del país. Es una muestra de cómo los principios liberales universalistas se convertirían en una suerte de ideología que legitima la exclusión de quienes no los comparten. Por eso algunos autores (Van Oers, 2014: 136) han llegado incluso a considerar comunitarista un test como el de Alemania, en la medida en que refleja la identidad liberal del país, a la que se espera que los nuevos ciudadanos se acomoden. Nos encontraríamos, así, ante lo que Joppke denomina, un «liberalismo represivo" (Joppke, 2007: 14-18), es decir, un liberalismo que "se transforma en identidad, en un modo de vida al que se espera que todo el mundo se amolde y que es desarrollado con una intención descaradamente excluyente contra quien es considerado como el «otro» del liberalismo: el islam y los musulmanes» (Joppke, 2010b: 2).

En cualquier caso, se ponen aquí de manifiesto una vez más las paradojas de la tolerancia liberal. Por una parte, desde ese giro hacia una comprensión de la identidad nacional y, en consecuencia, de la integración, como algo que gira en torno a valores cívicos -y, por tanto, universalistas-, cualquier forma de exclusión, en este caso, la exclusión de determinadas culturas por considerarlas no liberales ( $\mathrm{y}$ consecuentemente, no alemanas, no francesas) sería en sí misma, no alemana, no francesa, no acorde con los principios liberales, que son los que definen este país. El Estado liberal se encuentra, así, ante la tesitura de tener que tolerar prácticas iliberales o bien recurrir a medios iliberales para «liberarse de lo no liberal» y garantizar los valores liberales. Pero tanto tolerar prácticas iliberales como excluirlas por medios no liberales es algo no liberal. Y, en consecuencia, los países europeos estarían implementando políticas no liberales que violan los valores que pretenden proteger.

Por otra parte, la respuesta universalista a la cuestión de la integración plantea la dificultad de que los principios liberales no son rasgos específicos que definan a un país concreto frente a otro. Cabe preguntarse, entonces, en qué medida cumplen el objetivo de lograr la adhesión de los inmigrantes a esta sociedad particular, es decir, como

17. «Francia es una República indivisible, laica, democrática y social que garantiza la igualdad ante la ley de todos los ciudadanos sin distinción de origen, raza o religión y que respeta todas las creencias» (Constitución de 1958). 
señala Kymlicka, hasta qué punto «los valores políticos compartidos son suficientes para lograr la unidad social» (1995: 188).

Todo ello genera la duda de si los programas de integración cívica constituyen un método legítimo -incluso en su versión más universalista- y, al mismo tiempo, adecuado para probar la idoneidad de los candidatos a acceder a la ciudadanía. Comprobar la eficacia de este tipo de programas queda fuera de los límites de este estudio. Sin embargo, si, dado el contexto en el que surgen, todo parece apuntar a que un objetivo fundamental es lograr que los inmigrantes musulmanes se familiaricen con los valores y las instituciones liberales, no está clara su validez para evitar procesos de radicalización del tipo de los que se han manifestado recientemente en nuestro entorno. Pero, por supuesto, ir más allá de lo meramente cognitivo para exigir el compromiso interior de los inmigrantes con sus valores resulta totalmente ilegítimo desde los mismos supuestos de la democracia liberal. Y si lo que se pretende es lograr un conocimiento que les permita desenvolverse con éxito en la sociedad de acogida, quizá los exámenes y cursos deberían limitarse al conocimiento de "diversos aspectos de la vida cotidiana», como reza uno de los apartados del test en Alemania, o de «los aspectos económicos y sociales de la vida en Francia».

Finalmente, aunque se adoptan bajo la luz de una idea bidireccional de integración, todas las medidas analizadas aquí son de carácter unilateral o monológico. Es decir, constituyen variantes del modelo asimilacionista de integración en tanto que definen este proceso como asunción de rasgos culturales o políticos representativos de las sociedades de acogida, sin tratar de establecer cauces para que se produzca en esta una recepción de elementos de las comunidades foráneas. Esto es así incluso en el caso de Francia, donde el recurso a un contrato podría dar pie a implicar en la misma medida a las dos partes. Sin embargo, como hemos visto, a lo único que se compromete el Estado es a proporcionar esa atención inicial. Ciertamente, sería una asimilación en el sentido intransitivo que señala Brubaker (2001: 534), como un proceso que los inmigrantes realizan y no algo que la sociedad de acogida hace sobre ellos hasta lograr su completa absorción (la desaparición de las diferencias). Pero, por más que la integración cívica evite las consecuencias excluyentes de los modelos multiculturales y suavice las aristas coercitivas de los modelos clásicos de asimilación, no asume elementos de interculturalidad. Se halla, por tanto, bastante lejos del ajuste mutuo que se supone que es la integración y desde este punto de vista puede ser interpretada como un modelo particularista que se autoinstituye como universal.

\section{Referencias}

Assemblée Nationale. 2006. Loi n. 2006-911 du 24 juillet 2006 relative à l'immigration et à l'intégration. Disponible en : http://www.legifrance.gouv.fr/affichTexte. do?cidTexte=JORFTEXT000000266495 [consulta: 2 de diciembre de 2015].

Assemblée Nationale. 2011. Loi. 2011-672 du 16 juin 2011 relative à l'immigration, à l'intégration et à la nationalité. Disponible en: http://www.legifrance.gouv.fr/affi- 
chTexte.do?cidTexte=JORFTEXT000024191380\&dateTexte $=20151022$ [consulta: 2 de diciembre de 2015].

Assemblée Nationale. 2013. Décret n. 2013-794 du 30 août 2013 portant modification du décret $n^{\circ}$ 93-1362 du 30 décembre 1993 relatif aux déclarations de nationalité, aux décisions de naturalisation, de réintégration, de perte, de déchéance et de retrait de la nationalité française. 2013. Disponible en: http://www.legifrance.gouv.fr/affichTexte.do?cidTexte=JORFTEXT000027914456 [consulta: 2 de diciembre de 2015].

Ataman, Ferda. 2008. «Einbürgerung. Es darf keinen Gessinungstest geben!», Der Spiegel 14.06.2008. Disponible en: http://www.spiegel.de/politik/deutschland/ einbuergerung-es-darf-keinen-gesinnungstest-geben-a-559511.html [consulta: 22.04.2016].

Bauböck, Rainer y Christian Joppke. 2010. How liberal are citizenship tests? EUI Working Papers, 41. Robert Schuman Centre for Advanced Studies, EUDO Observatory. Disponible en: http://cadmus.eui.eu/bitstream/handle/1814/13956/RSCAS_2010_41 corr.pdf?sequence=3 [consulta: 21 de diciembre de 2015].

Beaujeu, Melodie. 2008. «Le modèle français d'intégration dans tous ses états: Entre reaffirmations républicaines et tentations populistes», International Journal on Multicultural Societies, 10 (1): 29-39.

Bertossi, Christophe y Abdellali Hajjat 2013. Country Report: France. EUDO Citizenship Observatory. Robert Schuman Centre for Advances Studies. Disponible en: http://eudo-citizenship.eu/admin/?p=file\&appl=countryProfiles\&f=France. pdf [consulta: 21 de diciembre de 2015].

Brubaker, Rogers. 1992. Citizenship and nationhood in France and Germany. Harvard: University Press.

Brubaker, Rogers. 2001. «The return of assimilation? Changing perspectives on immigration and its sequels in France, Germany and the United States», Ethnic and racial studies, 24 (4): 531-548. Disponible en: http://dx.doi.org/10.1080/01419870120049770 [consulta: 2 de diciembre de 2015].

Bundesministerium des Innern. 2008. Gesetz über den Aufenthalt, die Erwerbstätigkeit und die Integration von Ausländern im Bundesgebiet. 2008. Disponible en: http:// www.bmi.bund.de/SharedDocs/Gesetztexte/DE/A/AufenthG.html. [consulta: 2 de diciembre de 2015].

Bundesministerium des Innern. 2008. Gesetz zur Steuerung und Begrenzung der Zuwanderung und zur Regelung des Aufenthalts und der Integration von Unionsbügern und Ausländern. Disponible en: http://www.bmi.bund.de/SharedDocs/Gesetzestexte/DE/Zuwanderungsgesetz.pdf?_blob=publicationFile [consulta: 2 de diciembre de 2015].

Bundesministerium des Innern. 2008. Verordnung zu Einbürgerungstest und Einbürgerungskurs. Disponible en: http://www.gesetze-im-internet.de/einbtestv/ BJNR164900008.html [consulta: 2 de diciembre de 2015].

Carens, Joseph. 1987. «Aliens and citizens. The case for open borders», Review of Politics, 49 (2): 251-273. Disponible en: http://dx.doi.org/10.1017/S0034670500033817. 
Carens, Joseph. 2010. «The most liberal citizenship test is none at all», en Rainer Bauböck y Christian Joppke (eds.) How liberal are citizenship tests?. EUI Working Papers, 41. Robert Schuman Centre for Advanced Studies, EUDO Observatory. Disponible en: http://cadmus.eui.eu/bitstream/handle/1814/13956/RSCAS_2010_41corr.pdf?sequence=3: 19-20. [consulta: 21 de diciembre de 2015].

Carrera, Sergi y Elspeth Guild. 2010. "Are integration tests liberal? The 'universalistic liberal principles' as illiberal exceptionalism», en Rainer Bauböck y Christian Joppke (eds.), How liberal are citizenship tests? EUI Working Papers, 41. Robert Schuman Centre for Advanced Studies, EUDO Observatory. Disponible en: http://cadmus.eui.eu/bitstream/handle/1814/13956/RSCAS_2010_41corr.pdf?sequence=3: 29-34 [consulta: 21 de diciembre de 2015].

Consejo de Europa. 2008. Libro Blanco sobre el Diálogo Intercultural. «Vivir juntos con igual dignidad». Estrasburgo, 7 de mayo de 2008. Disponible en: http://www.coe. int/t/dg4/intercultural/Source/Pub_White_Paper/WhitePaper_ID_SpanishVersion.pdf [consulta: 28 de abril de 2016].

Consejo de la Unión Europea. 2004. Conclusiones del Consejo y de los Representantes de los Gobiernos de los Estados Miembros sobre los Principios Básicos Comunes de Integración. Consejo de Asuntos de Justicia e Interior, 19 de noviembre de 2004.

Deutsche Welle. 2006. "Requisito para nacionalizarse: el 'Muslim-Test'», Deutsche Welle 16.01.2006. Disponible en: http://www.dw.com/es/requisito-para-nacionalizarse-el-muslim-test/a-1858177 [consulta: 22 de abril de 2016].

Deutscher Bundestag. 2015. Staatsangehörigkeitsgesetz. 2015. Disponible en: http:// www.gesetze-im-internet.de/rustag/BJNR005830913.html [consulta: 2 de diciembre de 2015].

Die Zeit. 2010. «30 Fragen für den Pass», Die Zeit 11.01.2010. Disponible en: http:// www.zeit.de/online/2006/02/gesinnungstest [consulta: 22 de abril de 2016].

Favell, Adrien. 1995. Philosophies of Integration: Immigration and the Idea of Citizenship in France and Britain. London: Macmillan.

Gray, John. (2000). Las dos caras del liberalismo. Una interpretación de la tolerancia liberal. Barcelona: Paidós.

Habermas, Jürgen. 1996. Die Einbeziehung des Anderen. Frankfurt: Suhrkamp.

Hailbronner, Kay y Anuscheh Farahat. 2015. Country Report on Citizenship Law: Germany. EUDO Citizenship Observatory, Robert Schuman Centre for Advanced Studies. Disponible en: http://cadmus.eui.eu/bitstream/handle/1814/34478/EUDO_ CIT_2015_02-Germany.pdf?sequence=1 [consulta: 21 de diciembre de 2015].

Hajjat, Abdejalli. 2013. Naturalisation procedures for immigrants. France. EUDO Citizenship Observatory, Robert Schuman Centre for Advanced Studies. Disponible en: http://cadmus.eui.eu/bitstream/handle/1814/29782/NPR_2013_03-CITIMP-France.pdf?sequence $=1$ [consulta: 21 de diciembre de 2015].

Haut Conseil à l'Intégration. 2006. Le bilan de la politique d'intégration 2002-2005. Rapport au Premier Ministre. Paris: La documentation française. Disponible en : http://www.ladocumentationfrancaise.fr/var/storage/rapports-publics/064000272. pdf [consulta: 2 de diciembre de 2015]. 
Haut Conseil à l'Integration. 2003. Le contrat et l'intégration. Paris: La documentation francaise. Disponible en: http://www.ladocumentationfrancaise.fr/var/storage/rapports-publics/044000033.pdf [consulta: 2 de diciembre de 2015].

Innerarity, Carmen. 2009. «Comunidades de violencia: origen y significado de la violencia urbana en los barrios inmigrantes en las ciudades europeas», Anthropos: Huellas del conocimiento, 22: 169-189.

Innerarity, Carmen. 2007. «El Islam y la República: un conflicto entre dos identidades», Papers. Revista de Sociología, 84: 139-147.

Innerarity, Carmen. 2012. «La inclusión del otro en Francia y Alemania: el debate sobre el velo islámico», en Ignacio Sánchez de la Yncera y Marta Rodríguez Fouz (eds.), Dialécticas de la postsecularidad. Barcelona: Anthropos.

Innerarity, Carmen. 2013. «El debate sobre el velo islámico en Gran Bretaña. El multiculturalismo liberal y la identidad nacional», Revista de Estudios Políticos, 162: 149-174.

Innerarity, Carmen y Beatriz Acha. 2010. «Los discursos sobre ciudadanía e inmigración en Europa: universalismo, extremismo y educación", Politica y sociedad, 47: 63-84.

Jacobs, Dirk y Andrea Rea. 2007. «The End of National Models? Integration Courses and Citizenship Trajectories in Europe», International Journal on Multicultural Societies, 9 (2): 264-283.

Joppke, Christian y Ewa Morawska. 2003. Toward assimilation and citizenship. Immigrants in liberal nation-states, Basingstoke, UK: Palgrave Macmillan.

Joppke, Christian. 2007. «Beyond national models: civic integration policies for immigrants in Western Europe», West European Politics, 30: 1-22.

Joppke, Christian. 2008. «Immigration and the identity of citizenship: the paradox of universalism», Citizenship Studies, 12 (6): 533-546. Disponible en: http://dx.doi. org/10.1080/13621020802450445.

Joppke, Christian. 2009. Veil. Mirror of identity. Cambridge: Polity Press.

Joppke, Christian. 2010a. Citizenship and immigration. Cambridge: Polity Press.

Joppke, Christian. 2010b. «How liberal are citizenship tests?», en Rainer Bauböck y Christian Joppke (eds.), How liberal are citizenship tests? EUI Working Papers, 41. Robert Schuman Centre for Advanced Studies, EUDO Observatory. Disponible en: http://cadmus.eui.eu/bitstream/handle/1814/13956/RSCAS_2010_41corr. pdf?sequence=3: 1-4 and 39-41. [consulta: 21 de diciembre de 2015] .

Kastoryano, Riva. 2002. Negotiating identities. States and immigrants in France and Germany. Princeton: University Press.

Kostakopolou, Dora. 2010. "What liberalism is committed to and why current citizenship policies fail this test», en Rainer Bauböck y Christian Joppke (eds.), How liberal are citizenship tests? EUI Working Papers, 41. Robert Schuman Centre for Advanced Studies, EUDO Observatory. Disponible en: http://cadmus.eui.eu/ bitstream/handle/1814/13956/RSCAS_2010_41corr.pdf?sequence=3: 15-18. [consulta: 21 de diciembre de 2015]. 
Kymlicka, Will. 1995. Multicultural citizenship. A liberal theory of minority rights. Oxford: University Press.

Michalowski, Inés. 2011. «Required to assimilate? The content of citizenship tests in five countries», Citizenship Studies, 15 (6-7): 749-768. Disponible en: http://dx. doi.org/10.1080/13621025.2011.600116.

Ministére de l'Interieur. 2015. Le livret du citoyen. Disponible en : http://www.immigration.interieur.gouv.fr/Accueil-et-accompagnement/Le-livret-du-citoyen [consulta: 2 de diciembre de 2015).

Office français de l'immigration et de l'intégration. 2015. Préparer son intégration. Disponible en: http://www.ofii.fr/IMG/pdf/Preparer_son_integration.pdf [consulta: 2 de diciembre de 2015].

Orgad, Liav. 2010a. «Five liberal concerns about citizenship tests», en Rainer Bauböck y Christian Joppke (eds.), How liberal are citizenship tests? EUI Working Papers, 41. Robert Schuman Centre for Advanced Studies, EUDO Observatory. Disponible en: http://cadmus.eui.eu/bitstream/handle/1814/13956/RSCAS_2010_41corr.pdf?sequence=3: 21-24 [consulta: 21 de diciembre de 2015].

Orgad, Liav. 2010b. «Illiberal liberalism: cultural restrictions on migration and access to citizenship in Europe», American Journal of Comparative Law, 58 (1): 53-106. Disponible en: http://dx.doi.org/10.5131/ajcl.2009.0004.

Rawls, John. 1993: Political liberalism, New York: Columbia University Press.

Reimann, Anna. 2006. «'Muslim-Test': Liberaler Doppelpass im Bundestag», en Der Spiegel 19.01.2006. Disponible en: http://www.spiegel.de/politik/debatte/muslim-test-liberaler-doppelpass-im-bundestag-a-396185.html [consulta: 21 de abril de 2016].

Schnapper, Dominique. 2001. La comunidad de los ciudadanos: acerca de la idea moderna de nación. Madrid: Alianza.

Speckner, Kerstin. 2008. "Mach den Muslim-Test!», Die Tageszeitung 6.01.2006. Disponible en: http://www.taz.de/1/archiv/?dig=2006/01/06/a0124 [consulta: 21 de abril de 2016].

Staatsministerium Baden-Württemberg. 2005. Gesprächsleitfaden für die Einbürgerungsbehörden. Disponible en: http://www.baden-wuerttemberg.datenschutz. de/gesprachsleitfaden-fur-die-einburgerungsbehorden-stand-01-09-2005/. [consulta: 2 de diciembre de 2015].

Stasi, Bernard. 2003. Commission de réflexion sur l'application du principe de lä̈cité dans la République. Rapport au Président de la République. Paris: La Documentation française.

Van Oers, Ricky. 2014. Deserving citizenship. Citizenship tests in Germany, the Netherlands and the United Kingdom. Nijhoff: Leiden.

Wimmer, Andreas. 2002. Nationalist exclusion and ethnic conflict: Shadows of modernity. Cambridge: University Press. Disponible en: http://dx.doi.org/10.1017/ CBO9780511490415. 
Wolfrum, Rudiger y Volker Röben. 2006. Gutachten zur Vereinbarkeit des Gesprächsleitfaden für die Einbürgerungsbehörden des Landes Baden-Wü̈rttemberg mit Völkerrecht. Heidelberg.

Presentado para evaluación: 7 de enero de 2016.

Aceptado para publicación: 16 de junio de 2016.

\section{CARMEN INNERARITY}

carmen.innerarity@unavarra.es

Doctora en Filosofía y Letras por la Universidad de Navarra, con Premio Extraordinario de Doctorado. Como becaria del Gobierno alemán (Deutscher Akademischer Austauschdienst), amplió sus estudios en la Ludwig Maximiliam Universität (Munich) y la Westfälische Wilhelms Universität (Münster). Profesora titular de Sociología en la Universidad Pública de Navarra, donde ha desarrollado su actividad docente e investigadora desde 1995. Es autora de diversos trabajos sobre cultura política, conflictos de identidades, ciudadanía y multiculturalismo. En la actualidad sus investigaciones se centran en las políticas de gestión de la diferencia cultural en las sociedades europeas. 